\title{
Moving Seshadri Constants, and Coverings of Varieties of Maximal Albanese Dimension
}

\author{
Luca F. Di Cerbo \\ Luigi Lombardi* \\ University of Florida \\ University of Milan \\ Idicerbo@ufl.edu \\ luigi.lombardi@unimi.it
}

m

\begin{abstract}
Let $X$ be a smooth projective complex variety of maximal Albanese dimension, and let $L \rightarrow X$ be a big line bundle. We prove that the moving Seshadri constants of the pull-backs of $L$ to suitable finite abelian étale covers of $X$ are arbitrarily large. As an application, given any integer $k \geq 1$, there exists an abelian étale cover $p: X^{\prime} \rightarrow X$ such that the adjoint system $\left|K_{X^{\prime}}+p^{*} L\right|$ separates $k$-jets away from the augmented base locus of $p^{*} L$, and the exceptional locus of the pull-back of the Albanese map of $X$ under $p$.
\end{abstract}

*Partially supported by SIR 2014 AnHyC: "Analytic aspects in complex and hypercomplex geometry" (code RBSI14DYEB), Grant 261756 of the Research Council of Norway, and the Simons Foundation. 


\section{Contents}

1 Introduction 2

2 Moving Seshadri Constants and Base Loci 5

2.1 Augmented and Restricted Base Loci . . . . . . . . . . . . . 5

2.2 Moving Seshadri Constants . . . . . . . . . . . . . . . 7

3 Varieties of Maximal Albanese Dimension 9

3.1 Étale Covers Induced by the Albanese Variety . . . . . . . . . . 9

3.2 Convergence to the Universal Albanese Cover . . . . . . . . . . 10

3.3 Virtual Unboundedness of Moving Seshadri Constants . . . . . . 13

3.4 Positivity of Linear Systems . . . . . . . . . . . . . . . . 16

\section{Introduction}

The moving Seshadri constants $\varepsilon_{\text {mov }}(L ; x)$ of a big line bundle $L$ on a smooth projective variety are asymptotic invariants attached to the sequence $\left\{L^{\otimes m}\right\}_{m \geq 1}$ (cf. for instance [Nak02] and [ELMNP09]). They extend the definition and the geometric properties of the Seshadri constants $\varepsilon(L ; x)$ attached to nef line bundles, as introduced by Demailly in [Dem90], to the setting of big line bundles. For instance, analogously to $\varepsilon(L ; x)$, the moving Seshadri constants control the rates of growth of the orders of jets that are separated by the big line bundles $L^{\otimes m}$ at a point $x$ as a function of $m$.

In this paper, we prove that the moving Seshadri constants $\varepsilon_{\text {mov }}(L ; x)$ of a big line bundle on a smooth projective complex variety of maximal Albanese dimension are arbitrarily large on suitable abelian étale coverings of the variety. In other words, they are virtually unbounded, i.e, unbounded up to a sequence of étale covers. A similar problem was asked by Hwang ( $c f$. [Bauer et al.12, Problem 2.6.2]) in the setting of ample line bundles on smooth projective varieties with large algebraic fundamental group up to (not necessarily abelian) regular étale covers. Hwang's original problem was answered affirmatively in [DD19, Theorems 1.3 and 1.4], and furthermore generalized to the case of big and nef line bundles (cf. loc. cit. Theorem 1.7). Here we focus on varieties of maximal Albanese dimension, and we study this problem within the class of abelian étale covers induced by the Albanese map. The main result of this paper is the following. 
Theorem 1.1. Let $X$ be a smooth projective variety such that the Albanese map $\alpha: X \rightarrow \operatorname{Alb}(X)$ is generically finite onto its image, and let $L$ be a big line bundle on $X$. For any integer $N>0$, there exists an isogeny of abelian varieties $a: A^{\prime} \rightarrow \operatorname{Alb}(X)$ of finite degree together with a commutative diagram

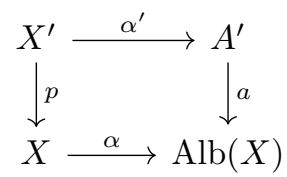

such that $\varepsilon_{\mathrm{mov}}\left(p^{*} L ; x\right) \geq N$ for any $x \notin p^{-1} \mathbf{B}_{+}(L) \cup \operatorname{Exc}\left(\alpha^{\prime}\right)$.

Here $\mathbf{B}_{+}(L)$ denotes the augmented base locus of $L$, while $\mathbf{E x c}\left(\alpha^{\prime}\right)$ stands for the exceptional locus of $\alpha^{\prime}$, i.e., the union of all positive-dimensional fibers. An ingredient of the proof of Theorem 1.1 is the fact that the augmented base locus of a big line bundle behaves well under pull-backs of finite morphisms. In order to show this fact, in Lemma 2.3 we will employ the description of $\mathbf{B}_{+}(L)$ in terms of currents, together with a result of Favre [Fav99] describing the local behavior of the pull-back of a current under a surjective map between smooth varieties.

We apply Theorem 1.1 in order to study the positivity of adjoint big line bundles on varieties of maximal Albanese dimension up to abelian étale covers. This is in the spirit of [HT99], [Yeu00] and [Wa05], where the authors study this problem for other classes of manifolds up to non-abelian regular étale covers.

Theorem 1.2. Let $X$ be a smooth projective variety such that the Albanese map $\alpha: X \rightarrow \operatorname{Alb}(X)$ is generically finite onto its image, and let $L$ be a big line bundle on $X$.

(i) There exists an étale cover $p: X^{\prime} \rightarrow X$ as in (1) such that for every $P \in \operatorname{Pic}^{0}\left(X^{\prime}\right)$ the linear series $\left|K_{X^{\prime}}+p^{*} L+P\right|$ is very ample away from $p^{-1} \mathbf{B}_{+}(L) \cup \operatorname{Exc}\left(\alpha^{\prime}\right)$.

(ii) For any integer $k \geq 0$, there exists an étale cover $p: X^{\prime} \rightarrow X$ as in (1) such that for every $P \in \mathrm{Pic}^{0}\left(X^{\prime}\right)$ the linear series $\left|K_{X^{\prime}}+p^{*} L+P\right|$ separates $k$-jets at any point $x \notin p^{-1} \mathbf{B}_{+}(L) \cup \mathbf{E x c}\left(\alpha^{\prime}\right)$.

Thus adjoint big line bundles on varieties of maximal Albanese dimension acquire more and more positivity on certain regular étale covers of large degree. In fact, for unconditional results concerning the same type of positivity, higher multiples of $K_{X}+L$ are needed. For instance, by [PP08, Theorem 5.8], the systems $\left|3\left(K_{X}+L\right)\right|$ are very ample away from $\operatorname{Exc}(\alpha)$ if $L$ is a big and nef line bundle on a smooth projective variety of maximal Albanese dimension.

When applied to the canonical bundle $L=K_{X}$ of a variety of general type, Theorem 1.2 assumes a slightly stronger formulation thanks to the main 
result of [BBP13]. In this way, we recover and extend [BPS19a, Theorem 4.1] by partly describing the locus where the system $\left|2 K_{X^{\prime}}+P\right|$ is very ample, or it separates $k$-jets.

Corollary 1.3. Let $X$ be a smooth projective variety of general type such that the Albanese map $\alpha: X \rightarrow \operatorname{Alb}(X)$ is generically finite onto its image.

(i) There exists an étale cover $p: X^{\prime} \rightarrow X$ as in (1) such that for every $P \in$ $\operatorname{Pic}^{0}\left(X^{\prime}\right)$ the linear series $\left|2 K_{X^{\prime}}+P\right|$ is very ample away from $\operatorname{Exc}\left(\alpha^{\prime}\right)$.

(ii) For any $k \geq 0$, there exists an étale cover $p: X^{\prime} \rightarrow X$ as in (1) such that for every $P \in \operatorname{Pic}^{0}\left(X^{\prime}\right)$ the linear series $\left|2 K_{X^{\prime}}+P\right|$ separates $k$-jets at any point $x \notin \operatorname{Exc}\left(\alpha^{\prime}\right)$.

For unconditional results, by [JLT13, Theorem A] we know that only the tri-canonical map of a variety of general type and maximal Albanese dimension is birational onto the image. Moreover, in [BLNP12, Theorem A], the authors characterize the primitive varieties (from the point of view of generic vanishing theory) of general type and maximal Albanese dimension for which the bi-canonical map is not birational ( $c f$. also [CCM-L98] for the case of surfaces). Other interesting results studying the positivity of linear systems on varieties of maximal Albanese dimension up to abelian étale covers are collected, among others, in [CJ18, Theorem 4.1], [LPS20, Theorem B], and [BPS19b, Theorem 3.7].

The techniques to establish Theorem 1.2 and Corollary 1.3, and hence Theorem 1.1, are somehow very different from the techniques employed in loc. cit., which rely, among other things, on generic vanishing theory, and the use of the eventual paracanonical map. Instead our arguments rely on the estimation of moving Seshadri constants along towers of coverings converging to the universal Albanese cover ( $c f$. §3.2), and the relationship occurring between the largeness of moving Seshadri constants and separation of jets. Our approach elaborates a circle of ideas developed in [DD19] for varieties with large fundamental group, whose roots lie in the fundamental work of J. Kollár [Kol93].

Throughout the paper, we work over the field of the complex numbers. At the same time, we wonder whether Theorem 1.1, or its corollaries, extends to positive characteristic. In this regard, the paper [Mur18] establishes the connection between the largeness of Seshadri constants and separation of jets of line bundles over any algebraically closed field.

\section{Acknowledgments}

We thank Rob Lazarsfeld and Christian Schnell for their interest in this work and fruitful conversations. We also thank Rita Pardini for pointing out the references [BPS19a], [BPS19b], and for constructive comments. LFDC thanks the 
Mathematics Department at Stony Brook University for the ideal research environment he enjoyed at the beginning of this research project. He also gratefully acknowledges the start up fund of the University of Florida for support during the final stages of this work. LL thanks the University of Florida and the Max Planck Institute for Mathematics in Bonn, for financial supports and excellent working conditions provided.

\section{Moving Seshadri Constants and Base Loci}

In this section, we describe the behavior of augmented and restricted base loci under finite maps. As an application, we extend [DD19, Theorem 1.7] to the setting of big line bundles and moving Seshadri constants. Throughout this section, we denote by $X$ a smooth projective variety, and by $D$ a $\mathbb{Q}$-divisor.

\subsection{Augmented and Restricted Base Loci}

We denote by $\operatorname{Bs}(m D)_{\text {red }}$ the base locus of the linear series $|m D|$ equipped with the reduced structure. The stable base locus of $D$ is the Zariski-closed set

$$
\mathbf{B}(D) \stackrel{\text { def }}{=} \bigcap_{m} \operatorname{Bs}(m D)_{\text {red }},
$$

where the intersection is over all positive integers $m$ such that $m D$ is integral.

Definition 2.1. (i) The augmented base locus of $D$ is the Zariski-closed set

$$
\mathbf{B}_{+}(D) \stackrel{\text { def }}{=} \bigcap_{A} \mathbf{B}(D-A)
$$

where the intersection is over all ample $\mathbb{Q}$-divisors $A$.

(ii) The restricted base locus of $D$ is defined as

$$
\mathbf{B}_{-}(D) \stackrel{\text { def }}{=} \bigcup_{A} \mathbf{B}(D+A),
$$

where the union is again over all ample $\mathbb{Q}$-divisors $A$.

Both $\mathbf{B}_{+}(D)$ and $\mathbf{B}_{-}(D)$ depend only on the numerical class of $D$. The locus $\mathbf{B}_{-}(D)$ is a countable union of closed sets whose closure is contained in $\mathbf{B}_{+}(D)$. The divisor $D$ is ample (resp. big) if and only if $\mathbf{B}_{+}(D)=\emptyset$ (resp. $\left.\mathbf{B}_{+}(D) \neq X\right)$. Moreover, $D$ is nef if and only if $\mathbf{B}_{-}(D)=\emptyset$. Finally, we note the series of inclusions $\mathbf{B}_{-}(D) \subseteq \mathbf{B}(D) \subseteq \mathbf{B}_{+}(D)$. We refer to [ELMNP06] for further properties about the augmented and restricted base loci. 
We state two lemmas concerning the behavior of the loci $\mathbf{B}_{+}(D)$ and $\mathbf{B}_{-}(D)$ under pull-backs. Let us begin with the restricted base locus (also called non-nef locus in [Bou04]).

Lemma 2.2. Let $p: X^{\prime} \rightarrow X$ be a surjective morphism of smooth projective varieties. If $L$ is a big line bundle on $X$, then $p^{-1} \mathbf{B}_{-}(L)=\mathbf{B}_{-}\left(p^{*} L\right)$.

Proof. The proof follows easily once we describe the restricted base locus of $L$ à la Boucksom [Bou04]. By Proposition 3.6 in [Bou04], given a big line bundle $L$ and a closed positive $(1,1)$-current with minimal singularities $T_{\min } \in[L]$, we have

$$
\mathbf{B}_{-}(L)=\left\{x \in X \mid \nu\left(T_{\min }, x\right)>0\right\},
$$

where $\nu\left(T_{\min }, x\right)$ denotes the Lelong number of $T_{\min }$ at the point $x \in X$. By standard compactness properties of positive currents, one can always find a current with minimal singularities in any big (or even pseudo-effective) cohomology class. Moreover, two currents with minimal singularities have the same Lelong numbers as they locally differ by a $(1,1)$-current of the form $\partial \bar{\partial} \varphi$ with $\varphi \in L^{\infty}$. For further details please refer to [Bou04, Section 2.8].

The pull-back $p^{*} T_{\min } \in\left[p^{*} L\right]$ of the current $T_{\min }$ is again a closed positive current with minimal singularities ( $c f$. [BEGZ10, Proposition 1.12]). Hence we have

$$
\mathbf{B}_{-}\left(p^{*} L\right)=\left\{x^{\prime} \in X^{\prime} \mid \nu\left(p^{*} T_{\min }, x^{\prime}\right)>0\right\} .
$$

At this point we employ the following local result of Favre [Fav99, Theorem 2 or Corollary 4]. If $f:\left(\mathbb{C}^{m}, 0\right) \rightarrow\left(\mathbb{C}^{n}, 0\right)$ is an holomorphic map generically of maximal rank equal to $\mathrm{n}$, then there exists a constant $C=C(f)>0$ depending only on $f$ such that

$$
\nu(T, 0) \leq \nu\left(f^{*} T, 0\right) \leq C \nu(T, 0)
$$

for any closed positive $(1,1)$-current $T$ on $\mathbb{C}^{n}$. As $p$ is holomorphic and surjective (and therefore of generic maximal rank), we conclude that

$$
\nu\left(p^{*} T_{\min }, x^{\prime}\right)>0 \Longleftrightarrow \nu\left(T_{\min }, p\left(x^{\prime}\right)\right)>0,
$$

for any $x^{\prime} \in X^{\prime}$.

By means of Lemma 2.2, we can show that the augmented base locus has a similar property under finite maps.

Lemma 2.3. Let $p: X^{\prime} \rightarrow X$ be a finite surjective morphism of smooth projective varieties. If $L$ is a big line bundle on $X$, then $p^{-1} \mathbf{B}_{+}(L)=\mathbf{B}_{+}\left(p^{*} L\right)$.

Proof. By [ELMNP06, Proposition 1.21], for all sufficiently small ample $\mathbb{Q}$ divisors $A$ on $X$ such that $L-A$ is a $\mathbb{Q}$-divisor, we have that

$$
\mathbf{B}_{-}(L-A)=\mathbf{B}_{+}(L-A)=\mathbf{B}_{+}(L) .
$$


Now, let $\left\{A_{i}\right\}$ be a sequence of such ample $\mathbb{Q}$-divisors converging to zero. Since the map $p: X^{\prime} \rightarrow X$ is finite, by Nakai-Moishezon criterion we know that the $\mathbb{Q}$-divisors in the sequence $\left\{p^{*} A_{i}\right\}$ are ample and clearly converging to zero. Thus, for $i$ large enough, we have

$$
\mathbf{B}_{-}\left(p^{*}\left(L-A_{i}\right)\right)=\mathbf{B}_{-}\left(p^{*} L-p^{*} A_{i}\right)=\mathbf{B}_{+}\left(p^{*} L-p^{*} A_{i}\right)=\mathbf{B}_{+}\left(p^{*} L\right) .
$$

Since the cone of big divisors is open, for $i$ big enough we have that $L-A_{i}$ is big. Note that Lemma 2.2 extends to $\mathbb{Q}$-divisors. Thus, for $i$ large enough, we have

$$
\mathbf{B}_{-}\left(p^{*}\left(L-A_{i}\right)\right)=p^{-1}\left(\mathbf{B}_{-}\left(L-A_{i}\right)\right)=p^{-1} \mathbf{B}_{+}\left(L-A_{i}\right)=p^{-1} \mathbf{B}_{+}(L) .
$$

By combining Equations (3) and (4), we obtain the desired identity.

Remark 2.4. In the case of big and nef line bundles, a different proof of Lemma 2.3 was given in [DD19, Lemma 4.1].

\subsection{Moving Seshadri Constants}

The moving Seshadri constant $\varepsilon_{\mathrm{mov}}(D ; x)$ is a measure of the local positivity of a big divisor. It agrees with the usual Seshadri constant $\varepsilon(D ; x)$ if the divisor $D$ is nef, and moreover it characterizes the augmented base locus $\mathbf{B}_{+}(D)$ as the set of points at which $\varepsilon_{\text {mov }}(D ; x)$ vanishes ( $c f$. [ELMNP09, Section 6]). In order to define $\varepsilon_{\text {mov }}(D ; x)$, we first recall the definition of the usual Seshadri constant.

The Seshadri constant of an integral nef divisor $F$ at a point $x \in X$ is the non-negative real number

$$
\varepsilon(F ; x) \stackrel{\text { def }}{=} \max \left\{\varepsilon \geq 0 \mid \mu^{*} F-\varepsilon E \text { is nef }\right\},
$$

where $\mu: \widetilde{X} \rightarrow X$ is the blow-up at $x$ with exceptional divisor $E$. By homogeneity, the definition of $\varepsilon(F ; x)$ extends to $\mathbb{Q}$-divisors.

Definition 2.5. Let $D$ be a $\mathbb{Q}$-divisor. The moving Seshadri constant of $D$ at $x \in X$ is

$$
\varepsilon_{\mathrm{mov}}(D ; x)= \begin{cases}\sup _{f^{*} D=A+E} \varepsilon(A ; x) & \text { if } x \notin \mathbf{B}_{+}(D) \\ 0 & \text { if } x \in \mathbf{B}_{+}(D)\end{cases}
$$

The supremum is taken over all projective morphisms $f: \widetilde{X} \rightarrow X$ with $\widetilde{X}$ smooth such that $f$ is an isomorphism near $x$, and all decompositions $f^{*} D=A+E$ where $A$ and $E$ are, respectively, an ample and effective $\mathbb{Q}$-divisors on $\widetilde{X}$ such that $f^{-1}(x)$ is not contained in the support of $E$.

Both quantities $\varepsilon(D ; x)$ and $\varepsilon_{\operatorname{mov}}(D ; x)$ only depend on the numerical equivalence class of $D$. We now need to recall two definitions from group theory. 
Definition 2.6. Let $\Gamma$ be a finitely generated group and $H$ be a subgroup. We say that $\Gamma$ is $H$-separable (or that $H$ is separable in $\Gamma$ ) if for every element $g \in \Gamma \backslash H$ there is a subgroup $K \subset \Gamma$ of finite index such that $H \subset K$ and $g \notin K$. Moreover, we say that $\Gamma$ is residually finite if the trivial subgroup $\operatorname{id}_{\Gamma}$ is separable in $\Gamma$.

We can now prove the main result this section. It extends [DD19, Theorem 1.7] to the case of big line bundles. We refer to [Kol93, Definition 1.7] for the definition of large fundamental group.

Theorem 2.7. Let $X$ be a smooth projective variety and $L$ a big line bundle. Assume that either the fundamental group $\pi_{1}(X)$ is residually finite and large, or the algebraic fundamental group $\pi_{1}^{\text {alg }}(X)$ is large. Then for any integer $N>0$, there exists an étale cover $p: X^{\prime} \rightarrow X$ such that $\varepsilon_{\operatorname{mov}}\left(p^{*} L ; x\right) \geq N$ for any $x \notin p^{-1} \mathbf{B}_{+}(L)=\mathbf{B}_{+}\left(p^{*} L\right)$.

Proof. By [DD19, Remark 1.5] it is enough to prove the theorem in the case $\pi_{1}^{\text {alg }}(X)$ is large. We consider the universal algebraic cover $\widehat{p}: \widehat{X} \rightarrow X$ associated to the kernel $\widehat{\Gamma}$ of the natural homomorphism $\pi_{1}(X) \rightarrow \pi_{1}^{\text {alg }}(X)$. As $\widehat{\Gamma}$ is separable in $\pi_{1}(X)$, there is a sequence $\left\{\Gamma_{j}\right\}_{j \geq 0}$ of nested finite index normal subgroups of $\pi_{1}(X)$ such that $\Gamma_{0}=\pi_{1}(X)$ and $\bigcap_{j \geq 0} \Gamma_{j}=\widehat{\Gamma}$. Consider the sequence

$$
\cdots \longrightarrow X_{j+1} \stackrel{p_{j+1}}{\longrightarrow} X_{j} \stackrel{p_{j}}{\longrightarrow} X_{j-1} \stackrel{p_{j-1}}{\longrightarrow} \cdots \stackrel{p_{1}}{\longrightarrow} X
$$

of regular coverings associated to $\left\{\Gamma_{j}\right\}_{j \geq 0}$.

Let $q_{k}: X_{k} \rightarrow X$ be the regular covering given by the composition $q_{k}=$ $p_{1} \circ \ldots \circ p_{k}$. Also, let $L \sim_{\mathbb{Q}} A+E$ be a decomposition of the big line bundle $L$ in $\mathbb{Q}$-divisors where $A$ is ample and $E$ effective. Moreover, let $N$ be an arbitrary positive integer. By [DD19, Theorem 1.3], there exists a positive integer $k=k(N, A)$ such that $\varepsilon\left(q_{j}^{*} A ; x\right) \geq N$ for any $j \geq k$. Thus, by the definition of moving Seshadri constant, we have

$$
\varepsilon_{\mathrm{mov}}\left(q_{j}^{*} L ; x\right) \geq \varepsilon\left(q_{j}^{*} A ; x\right) \geq N
$$

for any index $j \geq k$ and $x \notin q_{j}^{-1}(E)$.

Next, let $L \sim_{\mathbb{Q}} A_{i}+E_{i}$ be finitely many decompositions of $L$ in $\mathbb{Q}$-divisors with $A_{i}$ ample and $E_{i}$ effective as above, such that

$$
\mathbf{B}_{+}(L)=\bigcap_{i=1}^{l} \operatorname{Supp}\left(E_{i}\right)
$$

(cf. [ELMNP06, Definition 1.2 and Remark 1.3]). By the argument above, for any integer $i \in[1, l]$ we may find an integer $k_{i}$ such that

$$
\varepsilon_{\mathrm{mov}}\left(q_{j}^{*} L ; x\right) \geq \varepsilon\left(q_{j}^{*} A_{i} ; x\right) \geq N \quad \text { for any } \quad j \geq k_{i} \quad \text { and } \quad x \notin q_{j}^{-1}\left(E_{i}\right) .
$$


Define $K:=\max \left\{k_{1}, \ldots, k_{l}\right\}$. In view of Lemma 2.3, we observe that for any index $j \geq 1$ and $x \notin \mathbf{B}_{+}\left(q_{j}^{*} L\right)$ there exists an index $s_{j} \in[1, l]$ such that $x \notin$ $q_{j}^{-1}\left(E_{s_{j}}\right)$. Hence for any $j \geq K$ and $x \notin \mathbf{B}_{+}\left(q_{j}^{*} L\right)$, we compute

$$
\varepsilon_{\mathrm{mov}}\left(q_{j}^{*} L ; x\right) \geq \varepsilon\left(q_{j}^{*} A_{s_{j}} ; x\right) \geq N .
$$

\section{Varieties of Maximal Albanese Dimension}

In this section we prove Theorem 1.1.

\section{1 Étale Covers Induced by the Albanese Variety}

Let $X$ be a smooth projective variety of dimension $n$. The Albanese variety of $X$ is an abelian variety of dimension $g:=h^{0}\left(X, \Omega_{X}^{1}\right)$ defined as:

$$
A=\operatorname{Alb}(X)=H^{0}\left(X, \Omega_{X}^{1}\right)^{*} / H_{1}(X, \mathbb{Z})_{\text {t.f. }},
$$

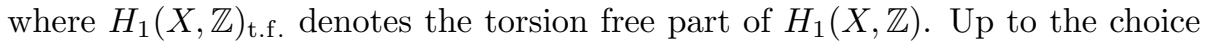
of a point, integration of holomorphic 1-forms on $X$ defines an Albanese map

$$
\alpha: X \longrightarrow A
$$

such that $\alpha^{*} H^{0}\left(A, \Omega_{A}^{1}\right)=H^{0}\left(X, \Omega_{X}^{1}\right)$. By the definition of the Albanese variety, there is an identification $\pi_{1}(A)=H_{1}(X, \mathbb{Z})_{\text {t.f. }}$ so that the natural homomorphism

$$
\alpha_{\#}: \pi_{1}(X) \longrightarrow \pi_{1}(A)
$$

is surjective. It follows that any irreducible abelian étale cover $\pi: B \rightarrow A$ of $A$ induces via the fiber product construction

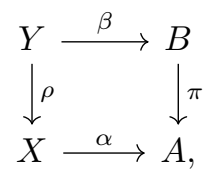

an étale cover $\rho: Y \rightarrow X$ of $X$ with the same properties. We refer to $Y$ (or $\rho$ ) as the pull-back of $B$ along $\alpha$.

Definition 3.1. Let $\Gamma$ be a residually finite group. We say that a sequence $\left\{\Gamma_{i}\right\}_{i \geq 0}$ of nested, normal, finite index subgroups of $\Gamma$ is a cofinal filtration if $\bigcap_{i=0}^{\infty} \bar{\Gamma}_{i}=\operatorname{id}_{\Gamma}$. 
Lemma 3.2. The group $\operatorname{ker}\left(\alpha_{\#}\right)$ is separable in $\pi_{1}(X)$. Moreover, for any tower of coverings

$$
\cdots \longrightarrow X_{i+1} \stackrel{s_{i+1}}{\longrightarrow} X_{i} \stackrel{s_{i}}{\longrightarrow} X_{i-1} \stackrel{s_{i-1}}{\longrightarrow} \cdots \stackrel{s_{1}}{\longrightarrow} X,
$$

obtained by pulling-back along $\alpha$ a tower of coverings

$$
\cdots \longrightarrow A_{i+1} \stackrel{r_{i+1}}{\longrightarrow} A_{i} \stackrel{r_{i}}{\longrightarrow} A_{i-1} \stackrel{r_{i-1}}{\longrightarrow} \cdots \stackrel{r_{1}}{\longrightarrow} A
$$

associated to a cofinal filtration of $\pi_{1}(A)$, we have

$$
\operatorname{ker}\left(\alpha_{\#}\right)=\bigcap_{i=1}^{\infty} \pi_{1}\left(X_{i}\right)
$$

Proof. We set

$$
\Gamma=\pi_{1}(X) \quad \text { and } \quad \Lambda=\pi_{1}(A) \simeq \mathbb{Z}^{2 g},
$$

so that $A=\mathbb{C}^{g} / \Lambda$. Let $\gamma \in \Gamma \backslash \operatorname{ker}\left(\alpha_{\#}\right)$ be an arbitrary element. Hence $\alpha_{\#}(\gamma) \neq$ $\operatorname{id}_{\Lambda}$, and there exists a finite index subgroup $\Lambda_{1} \leq \Lambda$ such that $\alpha_{\#}(\gamma) \notin \Lambda_{1}$. Here we are using the fact that finitely generated free Abelian groups are residually finite. Thus, let $a_{1}: A_{1} \rightarrow A$ be the étale cover associated to $\Lambda_{1}$, and let $s_{1}$ be the pull-back of $A_{1}$ along $\alpha$ :

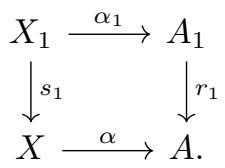

By standard covering space theory, we note the following equality of groups

$$
\left(s_{1}\right)_{\#}\left(\pi_{1}\left(X_{1}\right)\right)=\alpha_{\#}^{-1}\left(\left(r_{1}\right)_{\#} \pi_{1}\left(A_{1}\right)\right) .
$$

Hence the group $\Gamma_{1} \stackrel{\text { def }}{=} \pi_{1}\left(X_{1}\right)$ contains the subgroup $\alpha_{\#}^{-1}\left(\operatorname{id}_{\pi_{1}(A)}\right)=\operatorname{ker}\left(\alpha_{\#}\right)$ and avoids $\gamma$ by our choice of $\Lambda_{1}$. Moreover, the natural homomorphism

$$
\left(\alpha_{1}\right)_{\#}: \pi_{1}\left(X_{1}\right) \rightarrow \pi_{1}\left(A_{1}\right)
$$

is surjective as both $\left(s_{1}\right)_{\#}$ and $\left(r_{1}\right)_{\#}$ are injective homomorphism. By reiterating this process with $\alpha_{1}$ in place of $\alpha$, we generate a cofinal filtration $\left\{\pi_{1}\left(A_{i}\right)\right\}_{i=1}^{\infty}$ of $\pi_{1}(A)$ from which the lemma follows.

\subsection{Convergence to the Universal Albanese Cover}

We continue to denote by $X$ a smooth projective variety, and by $\alpha: X \rightarrow A$ the Albanese map. Moreover, we fix an ample line bundle $L$ on $X$ and denote by $\omega \in\left[c_{1}(L)\right]$ the induced smooth Kähler metric. Following Lemma 3.2, we set

$$
\Gamma=\Gamma_{0} \stackrel{\text { def }}{=} \pi_{1}(X) \quad \text { and } \quad \Gamma_{i} \stackrel{\text { def }}{=} \pi_{1}\left(X_{i}\right) \subset \Gamma .
$$


Furthermore, we denote by $\bar{q}_{i}: X_{i} \rightarrow X$ the composition $s_{1} \circ \ldots \circ s_{i}$ so that there is a commutative diagram

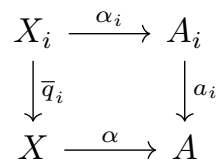

where $a_{i}=r_{1} \circ \ldots \circ r_{i}$. Finally, we denote by

$$
\bar{q}: \bar{X} \longrightarrow X
$$

the pull-back of the universal cover of $A$ along $\alpha$ :

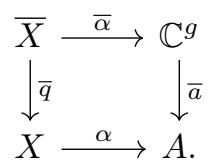

Throughout the paper, we refer to the cover $\bar{q}: \bar{X} \longrightarrow X$ defined in (6) as the universal Albanese cover of $X$. Notice that, up to a finite cover, the universal Albanese cover coincides with the universal Abelian cover of $X$. Indeed, these two infinite covers are the same if and only if $H_{1}(X, \mathbb{Z})_{\text {t.f. }}=H_{1}(X, \mathbb{Z})$.

Equivalently, the cover $\bar{q}$ can be defined as the regular covering associated to the separable normal subgroup $\operatorname{ker}\left(\alpha_{\#}\right) \triangleleft \pi_{1}(X)$. Hence, by Lemma 3.2, we have

$$
\bar{\Gamma} \stackrel{\text { def }}{=} \pi_{1}(\bar{X})=\operatorname{ker}\left(\alpha_{\#}\right)=\bigcap_{i \geq 1} \Gamma_{i}
$$

In Theorem 3.3, we will show that the sequence of covers $\bar{q}_{i}: X_{i} \rightarrow X$ converges to the universal Albanese cover in a precise way. First, we note that the coverings $X_{i}$ can be described as quotients of $\bar{X}$ :

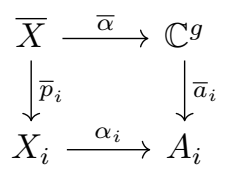

where

$$
X_{i} \simeq \bar{X} / \bar{\Gamma}_{i} \text { and } \quad \bar{\Gamma}_{i} \stackrel{\text { def }}{=} \Gamma_{i} / \bar{\Gamma}
$$

Secondly, we equip each $X_{i}$ with the Kähler metric $\bar{q}_{i}^{*} \omega$, and $\bar{X}$ with $\bar{q}^{*} \omega$. Finally, we define the following quantities

$$
\bar{r}_{i} \stackrel{\text { def }}{=} \inf \left\{d\left(z, \bar{\gamma}_{i} z\right) \mid z \in \bar{X}, \quad \bar{\gamma}_{i} \in \bar{\Gamma}_{i}, \quad \bar{\gamma}_{i} \neq \operatorname{id}_{\bar{\Gamma}_{i}}\right\},
$$

where the distance $d(-,-)$ is measured with respect to the metric $\bar{q}^{*} \omega$ on $\bar{X}$. 
Theorem 3.3. Let

$$
\bar{p}_{i}:\left(\bar{X}, \bar{q}^{*} \omega\right) \longrightarrow\left(X_{i}, \bar{q}_{i}^{*} \omega\right)
$$

be the Riemannian covering maps induced by the inclusions $\bar{\Gamma}_{i} \subset \bar{\Gamma}_{0}$ as in (7). Then, for any $z \in \bar{X}$, the maps

$$
\bar{p}_{i}: B\left(z ; \frac{\bar{r}_{i}}{2}\right) \longrightarrow \bar{p}_{i}\left(B\left(z ; \frac{\bar{r}_{i}}{2}\right)\right)
$$

are isometries and

$$
\lim _{i \rightarrow \infty} \bar{r}_{i}=\infty
$$

Proof. By definition of the numerical invariant $\bar{r}_{i}$, the map $\bar{p}_{i}: B\left(z ; \frac{\bar{r}_{i}}{2}\right) \rightarrow$ $\bar{p}_{i}\left(B\left(z ; \frac{\bar{r}_{i}}{2}\right)\right)$ is a biholomorphism for any $z \in \bar{X}$, and since we are pulling back the metric $\omega$ on $X$, it is an isometry.

Now we prove (9). We proceed by contradiction and assume that there exist a positive constant $M$, and infinite sequences $\left\{z_{i}\right\} \subset \bar{X}$ and $\left\{\bar{\gamma}_{i}\right\} \subset \bar{\Gamma}_{i}$, such that $d\left(z_{i}, \bar{\gamma}_{i} z_{i}\right) \leq 2 M$ and $\bar{\gamma}_{i} \neq \mathrm{id}_{\bar{\Gamma}_{i}}$. Let $D$ be a fundamental domain for $X$ in $\bar{X}$. Thus, $D \subset \bar{X}$ is a connected open set such that $\bar{q}: D \rightarrow X$ is injective and $\bar{q}: \bar{D} \rightarrow X$ is surjective, where $\bar{D}$ is the closure of $D$ in $\bar{X}$. Thus, for any $i$, there exists an element $g_{i} \in \bar{\Gamma}_{0}$ such that $g_{i} z_{i} \in \bar{D}$. Let us define $z_{i}^{\prime}=g_{i} z_{i}$ and $\bar{\gamma}_{i}^{\prime}=g_{i} \bar{\gamma}_{i} g_{i}^{-1}$. Since $\bar{\Gamma}_{i}$ is a normal subgroup of $\bar{\Gamma}_{0}$, we have that $\bar{\gamma}_{i}^{\prime} \in \bar{\Gamma}_{i}$. By compactness of $\bar{D}$, there exists a subsequence $\left\{z_{i_{j}}^{\prime}\right\}$ converging to a point $\bar{z} \in \bar{D}$. Now since

$$
d\left(z_{i}^{\prime}, \bar{\gamma}_{i}^{\prime} z_{i}^{\prime}\right)=d\left(g_{i} z_{i}, g_{i} \bar{\gamma}_{i} z_{i}\right)=d\left(z_{i}, \bar{\gamma}_{i} z_{i}\right)
$$

we have that

$$
d\left(\bar{z}, \bar{\gamma}_{i_{j}}^{\prime} \bar{z}\right) \leq d\left(\bar{z}, z_{i_{j}}^{\prime}\right)+2 M .
$$

Since $d\left(\bar{z}, z_{i_{j}}^{\prime}\right) \rightarrow 0$, we then conclude that, up to a subsequence, $\bar{\gamma}_{i_{j}}^{\prime} \bar{z}$ converges to a point $w \in B(\bar{z} ; 2 M+\varepsilon)$ for some $\varepsilon>0$. This implies that

$$
\bar{q}(\bar{z})=\bar{q}\left(\bar{\gamma}_{i_{j}}^{\prime} \bar{z}\right) \longrightarrow \bar{q}(w) .
$$

Thus, there exists $\bar{\gamma} \in \bar{\Gamma}_{0}$ such that $\bar{\gamma} w=\bar{z}$. We therefore conclude

$$
\left(\bar{\gamma}_{i_{j}}^{\prime} \cdot \bar{\gamma}\right) w=\bar{\gamma}_{i_{j}}^{\prime} \bar{z} \longrightarrow w
$$

Now the action of $\bar{\Gamma}_{0}$ on $\bar{X}$ is properly discontinuous, so that $\bar{\gamma}_{i_{j}}^{\prime} \cdot \bar{\gamma}=\operatorname{id}_{\bar{\Gamma}_{i_{j}}}$ for all $j$ sufficiently large. Thus, we must have $\bar{\gamma}=\mathrm{id}_{\bar{\Gamma}_{0}}$ since $\bigcap \bar{\Gamma}_{i_{j}}=\mathrm{id}_{\bar{\Gamma}_{0}}$, which then implies the contradiction $\bar{\gamma}_{i_{j}}^{\prime}=\mathrm{id}_{\bar{\Gamma}_{i_{j}}}$. 


\subsection{Virtual Unboundedness of Moving Seshadri Constants}

We keep notation as in the previous subsection. In addition, we assume that $X$ is of maximal Albanese dimension, namely that the Albanese map

$$
\alpha: X \longrightarrow A
$$

is generically finite onto the image. We denote by $\operatorname{Exc}(-)$ the exceptional locus of a morphism that is generically finite onto its image, i.e., the union of all its positive-dimensional fibers.

Lemma 3.4. For any integer $N>0$, there exists a positive integer $k(N)$ such that

$$
\left(\left(\bar{q}_{i}^{*} L\right)^{\operatorname{dim} Z} \cdot Z\right) \geq N
$$

for any $i \geq k(N)$ and irreducible subvariety $Z \subset X_{i}$ not entirely contained in $\operatorname{Exc}\left(\alpha_{i}\right)$.

Proof. We claim that for any integer $i$, and irreducible subvariety $Z \subset X_{i}$ not entirely contained in $\operatorname{Exc}\left(\alpha_{i}\right)$, we must have $Z \subsetneq \bar{p}_{i}\left(B\left(z ; \frac{\bar{r}_{i}}{2}\right)\right)$ for any ball $B\left(z ; \frac{\bar{r}_{i}}{2}\right)$ in $\bar{X}$. We proceed by contradiction and assume that this is not the case. By Theorem 3.3, we can then find a copy of $Z$ inside $\bar{X}$. By considering the commutative diagram

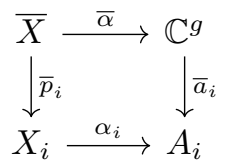

we have that $Z$ inside $\bar{X}$ is not entirely contained $\operatorname{Exc}(\bar{\alpha})$. Thus, $\bar{\alpha}(Z)$ is a compact analytic subvariety of positive dimension inside $\mathbb{C}^{g}$ which is impossible.

Next, we observe that all metrics $\bar{q}_{i}^{*} \omega$ have uniformly bounded geometry. In fact, they are pull backs of a fixed smooth Kähler metric on the compact manifold $X$ via étale covers. In particular, there exist positive constants $r=$ $r(L), C_{1}, C_{2}$ such that

$$
C_{1} \omega_{E} \leq \bar{q}^{*} \omega \leq C_{2} \omega_{E}
$$

on any ball $B(z ; r) \subset \bar{X}$ (here $\omega_{E}$ denotes the standard Euclidean Kähler metric on $\left.\mathbb{C}^{g}\right)$. Moreover, we can arrange (10) to hold true for any $\bar{q}_{i}^{*} \omega$, as well as for $\bar{q}^{*} \omega$ on balls of the same size. Thus, for any $i$, given any irreducible subvariety $Z \subset X_{i}$ of pure dimension $l$, there exists a positive constant $K_{l}=K\left(r, C_{1}, C_{2} ; l\right)$ such that for any point $p \in Z$ we have

$$
\operatorname{Vol}_{l}(B(p ; r) \cap Z) \geq K_{l} .
$$

Here the volume is computed by the integral of the $l$-th power of $\bar{q}_{i}^{*} \omega$ over the smooth part of $Z$. The inequality (11) follows from the inequalities (10) and the 
same statement for the euclidean metric on $\mathbb{C}^{g}$, see for example [DD19, Remark 3.5]. Recall now that for any $i$, given a point $p \in Z$, the subvariety $Z$ is not entirely contained in $B\left(p ; \frac{\bar{r}_{i}}{2}\right)$. Thus we have that

$$
\operatorname{Vol}_{l}(Z) \geq \alpha_{l} \bar{r}_{i}
$$

for some constant $\alpha_{l}=\alpha\left(K_{l}\right)>0$. Next, let us observe that by construction we have

$$
\left(\bar{q}_{i}^{*} L\right)^{l} \cdot Z=\operatorname{Vol}_{l}(Z) \geq \alpha_{l} \bar{r}_{i}
$$

for any $i$. By Theorem 3.3, we have that $\bar{r}_{i} \rightarrow \infty$ and the lemma follows.

We apply the previous result in order to show that the Seshadri constants of pull-backs of ample line bundles under étale covers coming from the Albanese variety are unbounded. In other words, they are virtually unbounded in the class of finite abelian covers induced by the Albanese map.

Lemma 3.5. For any integer $N>0$, there exists a positive integer $k(N)$ such that $\varepsilon\left(\bar{q}_{i}^{*} L ; x\right) \geq N$ for any $i \geq k(N)$ and $x \in X_{i} \backslash \operatorname{Exc}\left(\alpha_{i}\right)$.

Proof. Let $n$ be the dimension of $X$ and let $m=m(L)$ be a positive integer such that $m L-K_{X}$ is ample and $m L-2 K_{X}$ is very ample. We define $L^{\prime}=m L-K_{X}$. By Anghern-Siu Theorem [AS95] the line bundle $K_{X}+m_{0} L^{\prime}$ is base point free for all $m_{0} \geq\left(\begin{array}{c}n+1 \\ 2\end{array}\right)+1$. We therefore have that

$$
K_{X}+\left(\left(\begin{array}{c}
n+1 \\
2
\end{array}\right)+1\right) L^{\prime}+m L-2 K_{X}=\left(\left(\begin{array}{c}
n+1 \\
2
\end{array}\right)+2\right) L^{\prime}
$$

is base point free. Concluding, $m_{0} L^{\prime}$ is base point free for any $m_{0} \geq\left(\begin{array}{c}n+1 \\ 2\end{array}\right)+2$. Now define the constant

$$
N^{\prime} \stackrel{\text { def }}{=} \max _{c=0, \ldots, n}\left\{\left(1+\left(\begin{array}{c}
n+1 \\
2
\end{array}\right)(n-c)+\frac{n !}{c !}\right)^{c}(n+s)^{n}+1\right\}
$$

where $s>0$ is a fixed integer to be determined later. By Proposition 3.4, there exists an integer $i_{0}=i_{0}(s)$ such that

$$
\left(\left(\bar{q}_{i}^{*} L^{\prime}\right)^{\operatorname{dim} V} \cdot V\right) \geq N^{\prime}
$$

for any $i \geq i_{0}$ and any subvariety $V \subseteq X_{i}$ not entirely contained in $\operatorname{Exc}\left(\bar{\alpha}_{i}\right)$. Moreover, by construction, we have that the line bundle

$$
\bar{q}_{i}^{*}\left(L^{\prime}-K_{X}\right)=\bar{q}_{i}^{*} L^{\prime}-K_{X_{i}}
$$

is nef and

$$
\bar{q}_{i}^{*}\left(m_{0} L^{\prime}\right)
$$


is base point free. By a theorem of Ein-Lazarsfeld-Nakamaye [ELN96, Theorem 4.4], we know that for any $x \in X_{i} \backslash \operatorname{Exc}\left(\bar{\alpha}_{i}\right)$ the linear system $\left|K_{X_{i}}+\bar{q}_{i}^{*} L^{\prime}\right|$ separates $s$-jets at $x$. Since

$$
K_{X_{i}}+\bar{q}_{i}^{*} L^{\prime}=m \bar{q}_{i}^{*} L
$$

by [Dem90, Proposition 6.3] we conclude that $\varepsilon\left(\bar{q}_{i}^{*} L ; x\right) \geq \frac{s}{m}$. Thus, given any $N>0$, it suffices to take an integer $s=s(N) \geq 0$ such that $\frac{s}{m} \geq N$. We point out that the positive integer $m$ depends only on the line bundle $L$. Given this choice for $s$, for any $i \geq i_{0}(s)$ the associated cover $\bar{q}_{i}: X_{i} \rightarrow X$ satisfies the conclusion of the theorem.

We can now prove the main theorem stated in the Introduction.

Proof of Theorem 1.1. Let

$$
L \sim_{\mathbb{Q}} A+E
$$

be a decomposition of the big line bundle $L$ in $\mathbb{Q}$-divisors with $A$ ample and $E$ effective. By Lemma 3.5, given any positive constant $N$, there exists a positive integer $k=k(N, A)$ such that $\varepsilon\left(\bar{q}_{j}^{*} A ; x\right) \geq N$ for any $x \notin \operatorname{Exc}\left(\alpha_{j}\right)$ and $j \geq k$. Thus, by the definition of moving Seshadri constant, we have

$$
\varepsilon_{\mathrm{mov}}\left(\bar{q}_{j}^{*} L ; x\right) \geq \varepsilon\left(\bar{q}_{j}^{*} A ; x\right) \geq N
$$

for any index $j \geq k$ and $x \notin \bar{q}_{j}^{*}(E) \cup \mathbf{E x c}\left(\alpha_{j}\right)$.

Next, let $L \sim_{\mathbb{Q}} A_{i}+E_{i}$ be finitely many decompositions of $L$ in $\mathbb{Q}$-divisors with $A_{i}$ ample and $E_{i}$ effective, as above, satisfying

$$
\mathbf{B}_{+}(L)=\bigcap_{i=1}^{l} \operatorname{Supp}\left(E_{i}\right)
$$

By the above argument, for any integer $i \in[1, l]$ we may find an integer $k_{i}$ such that

$$
\varepsilon_{\mathrm{mov}}\left(\bar{q}_{j}^{*} L ; x\right) \geq \varepsilon\left(\bar{q}_{j}^{*} A_{i} ; x\right) \geq N \text { for any } x \notin \bar{q}_{j}^{*}\left(E_{i}\right) \cup \operatorname{Exc}\left(\alpha_{j}\right) \text { and } j \geq k_{i} .
$$

Define $K:=\max \left\{k_{1}, \ldots, k_{l}\right\}$. In view of Lemma 2.3, we observe that for any index $j \geq 1$ and $x \notin \mathbf{B}_{+}\left(\bar{q}_{j}^{*} L\right)$ there exists an index $s_{j} \in[1, l]$ such that $x \notin$ $\bar{q}_{j}^{-1}\left(E_{i_{j}}\right)$. Finally, fix any index $j \geq K$. Hence, for any $x \notin \mathbf{B}_{+}\left(\bar{q}_{j}^{*} L\right) \cup \operatorname{Exc}\left(\alpha_{j}\right)$ we have

$$
\varepsilon_{\mathrm{mov}}\left(\bar{q}_{j}^{*} L ; x\right) \geq \varepsilon\left(\bar{q}_{j}^{*} A_{s_{j}} ; x\right) \geq N .
$$




\subsection{Positivity of Linear Systems}

A linear system $|L|$ on a smooth projective variety $X$ is said to separate $k$-jets at a point $x \in X$ if the natural homomorphism

$$
H^{0}(X, L) \longrightarrow H^{0}\left(X, L \otimes \mathcal{O}_{X} / \mathfrak{m}_{x}^{k+1}\right)
$$

is surjective. In order to prove Theorem 1.2 and Corollary 1.3 of the Introduction, we need to establish the connection between moving Seshadri constants and separation of jets of adjoint line bundles.

Proposition 3.6. Let $X$ be a smooth projective variety of dimension $n$ and $L$ a big line bundle.

(i) If $\varepsilon_{\mathrm{mov}}(L ; x)>n+k$, then $\left|K_{X}+L\right|$ separates $k$-jets at $x$.

(ii) Let $V \subsetneq X$ be a Zariski-closed subset. If $\varepsilon_{\mathrm{mov}}(L ; x)>2 n$ for all $x \notin V$, then $\left|K_{X}+L\right|$ is very ample away from $V$.

We refer to [ELMNP09, Proposition 6.8] for the proof of (i). Point (ii) follows from the proof of point $(i)$ by employing the main idea of [Laz04, Proposition 5.1.19 (ii)].

Proof of Theorem 1.2. Set $n=\operatorname{dim} X$ and let $N$ be an arbitrary positive integer. Note that if $D$ is a divisor, then $\mathbf{B}_{+}(D)=\mathbf{B}_{+}(D+P)$ and $\varepsilon_{\text {mov }}(D ; x)=$ $\varepsilon_{\text {mov }}(D+P ; x)$ for any divisor $P$ with $c_{1}(P)=0$. Hence, by Theorem 1.1, there exists a commutative diagram as in (1) such that

$$
\varepsilon_{\mathrm{mov}}\left(p^{*} L+P ; x\right)=\varepsilon_{\mathrm{mov}}\left(p^{*} L ; x\right)>N
$$

for any $x \notin p^{-1} \mathbf{B}_{+}(L) \cup \mathbf{E x c}\left(\alpha^{\prime}\right)$ and $P \in \operatorname{Pic}^{0}\left(X^{\prime}\right)$. The corollary follows by Proposition 3.6.

Proof of Corollary 1.3. We set $L=K_{X}$ in Theorem 1.2, and observe that, by [BBP13], the augmented base locus $\mathbf{B}_{+}\left(K_{X^{\prime}}\right)$ of a canonical divisor of a variety of general type is uniruled. This yields the inclusion

$$
\mathbf{B}_{+}\left(K_{X^{\prime}}\right) \subseteq \mathbf{E x c}\left(\alpha^{\prime}\right)
$$

since any morphism from a smooth variety to an abelian variety contracts all rational curves. The proof is complete.

Finally, we refine Corollary 1.3 in the case of varieties with finite Albanese map. In this setting, the result assumes the strongest possible formulation. 
Corollary 3.7. Let $X$ be a smooth projective variety such that the Albanese map $\alpha: X \rightarrow A$ is finite onto the image.

(i) If $X$ is of general type, then there exists a commutative diagram as in (1) such that for every $P \in \operatorname{Pic}^{0}\left(X^{\prime}\right)$ the linear system $\left|2 K_{X^{\prime}}+P\right|$ defines an embedding of $X^{\prime}$ into a projective space.

(ii) There exists a commutative diagram as in (1) such that the linear system $\left|2 K_{X^{\prime}}\right|$ induces the Iitaka fibration.

Proof. The first point is application of Theorem 1.2 as $\operatorname{Exc}\left(\alpha^{\prime}\right)=\emptyset$. For the second point, we set $n=\operatorname{dim} X$. By [Kaw81, Theorem 13], we can find an étale cover $X_{1} \times B \rightarrow X$ such that $X_{1}$ is a variety of general type of dimension $k=\operatorname{kod}(X)$, and $B$ is an abelian variety of dimension $n-k$. By point $(i)$, there exists an étale cover $X_{1}^{\prime} \rightarrow X_{1}$ such that the map associated to the linear system $\left|2 K_{X_{1}^{\prime}}\right|$ is an embedding. Now, the étale cover $X^{\prime}:=X_{1}^{\prime} \times B \rightarrow X$ is such that the linear system $\left|2 K_{X^{\prime}}\right|$ induces the Iitaka fibration.

\section{References}

[AS95] U. Angehrn, Y.-T. Siu, Effective freeness and point separation for adjoint bundles. Invent. Math. 122 (1995), no. 2, 291-308

[BPS19a] M. A. Barja, R. Pardini, L. Stoppino, The eventual paracanonical map of a variety of maximal Albanese dimension. Alg. Geom. 6 (2019), no. 3, 302-311.

[BPS19b] M. A. Barja, R. Pardini, L. Stoppino, Linear systems on irregular varieties. J. Inst. Math. Jussieu (2019), 139.

[Bauer et al.12] T. Bauer, C. Bocci, S. Cooper, S. Di Rocco, M. Dumnicki, B. Harbourne, K. Jabbusch, A. L. Knutsen, A. Küronya, R. Miranda, J. Roé, H. Schenck, T. Szemberg, Z. Teitler, Recent developments and open problems in linear series. Contributions to algebraic geometry, pp. 93-140, EMS Ser. Congr. Rep., Eur. Math. Soc., Zürich, 2012

[BBP13] S. Boucksom, A. Broustet, G. Pacienza, Uniruledness of stable base loci of adjoint linear systems via Mori theory. Math. Z. 275 (2013), no. 1-2, 499-507

[BEGZ10] S. Boucksom, P. Eyssidieux, V. Guedj, A. Zeriahi, Monge-Ampère equations in big cohomology classes. Acta Math. 205 (2010), no. 2, 199-262 
[BLNP12] M. A. Barja, M. Lahoz, J. C. Naranjo, G. Pareschi, On the bicanonical map of irregular varieties. J. Algebraic Geom. 21 (2012), no. $3,445-471$

[Bou04] S. Boucksom, Divisorial Zariski decomposition on compact complex manifolds. Ann. Sci. École Norm. Sup., (4) 37 (2004), no. 1, 45-76

[CCM-L98] F. Catanese, C. Ciliberto, M. Mendes Lopes, On the classification of irregular surfaces of general type with nonbirational bicanonical map. Trans. Amer. Math. Soc. 350 (1998), no. 1, 275-308

[CJ18] J. A. Chen, Z. Jiang, Positivity in varieties of maximal Albanese dimension. J. Reine Angew. Math. 736 (2018), 225-253

[Dem90] J.-P. Demailly, Singular Hermitian metrics on positive line bundles, Complex Algebraic Varieties (Bayreuth, 1990), 87-104, Lect. Notes in Math., 1507, Springer, Berlin, 1992

[DD19] G. Di Cerbo, L. F. Di Cerbo, On Seshadri constants of varieties with large fundamental group. Ann. Sc. Norm. Super. Pisa Cl. Sci. (5) 19 (2019), no. 1, 335-344.

[ELN96] L. Ein, R. Lazarsfeld, M. Nakamaye, Zero-estimates, intersection theory, and a theorem of Demailly. Higher-dimensional complex varieties (Trento, 1994), 183-207, de Gruyter, Berlin, 1996

[ELMNP06] L. Ein, R. Lazarsfeld, M. Mustata, M. Nakamaye, M. Popa, Asymptotic invariants of base loci. Ann. Inst. Fourier (Grenoble) 56 (2006), no. 6, 1701-1734

[ELMNP09] L. Ein, R. Lazarsfeld, M. Mustata, M. Nakamaye, M. Popa, Restricted volumes and base loci of linear series. Amer. J. Math. 131 (2009), no. 3, 607-651

[Fav99] C. Favre, Note on pull-back and Lelong number of currents. Bull. Soc. Math. France 127 (1999), no. 3, 445-458

[HT99] J.-M. Hwang, W.-K. To. On Seshadri constants of canonical bundles of compact complex hyperbolic spaces. Compositio Math. 118 (1999), 203-215

[JLT13] Z. Jiang, M. Lahoz, S. Tirabassi. On the Iitaka fibration of varieties of maximal Albanese dimension. Int. Math. Res. Not. IMRN (2013), no. 13, 2984-3005

[Kaw81] Y. Kawamata, Characterization of Abelian Varieties. Compositio Math. 43 (1981), no. 2, 253-276

[Kol93] J. Kollár, Shafarevich maps and plurigenera of algebraic varieties. Invent. Math. 113 (1993), no. 1, 177-215 
[Laz04] R. Lazarsfeld, Positivity in algebraic geometry I, Ergebnisse der Mathematik und ihrer Grenzgebiete, vol. 48, Berlin: Springer 2004

[LPS20] L. Lombardi, M. Popa, C. Schnell, Pushforwards of pluricanonical bundles under morphisms to abelian varieties. J. Eur. Math. Soc. 22 (2020), no. 8, 2511-2536

[Mur18] T. Murayama, Frobenius-Seshadri constants and characterizations of projective space. Math. Res. Lett. 25 (2018), no. 3, 905-936

[Nak02] M. Nakamaye. Base loci of linear series are numerically determined. Trans. Amer. Math. Soc. 355 (2002), no. 2, 551-566

[PP08] G. Pareschi, M. Popa, $M$-regularity and the Fourier-Mukai transform, Pure Appl. Math. Q. 4 (2008), 587-611

[Yeu00] S.-K. Yeung, Very ampleness of line bundles and canonical embeddings of coverings of manifolds. Compositio Math. 123 (2000), no. $2,209-223$

[Wa05] X. Wang, Effective very ampleness of the canonical line bundles on ball quotients. J. Geom. Anal. 25 (2015), no. 2, 740-760 